\title{
LIMIT-OPERATORS AND TOPOLOGICAL COREFLECTIONS
}

\author{
BY \\ HORST HERRLICH
}

Recently the categorial notions of epireflective and mono-coreflective subcategories have turned out to be useful tools in describing and analyzing certain seemingly typical topological situations. Whereas the concept of topological epireflections is closely related to embedding-problems the dual concept of topological coreflections (which are automatically mono-coreflections) is closely related to problems of modification of topologies on fixed underlying sets and, as will be seen in this paper, to the concept of limit-operators. Whereas examples of topological epireflections are abundant this is not so obvious for coreflections. It is the aim of this paper to provide us with a sufficient supply of topological coreflections.

In $\S I$ general methods are described concerning how to obtain topological coreflections and relations are analysed between topological coreflections and limit-operators. In §II the lattice of all topological coreflections is investigated.

I. We consider only subcategories $\mathfrak{A}$ of the category $\mathfrak{T}$ of topological spaces which are full( $\left.{ }^{1}\right)$ and replete $\left({ }^{2}\right)$ so that there is no essential difference between subcategories of $\mathfrak{I}$, classes of topological spaces which contain with every space $X$ each space homeomorphic to $X$, and topological properties. We assume further that $\mathfrak{A}$ contains at least one nonempty space.

$\mathfrak{U}$ is called coreflective in $\mathfrak{T}$ iff for every space $X$ there exists a space $X_{\mathfrak{A}}$ in $\mathfrak{A}$ and a map $\left(^{3}\right) C_{\mathfrak{Q}}: X_{\mathfrak{U}} \rightarrow X$ such that for any space $A$ in $\mathfrak{A}$ and any map $f: A \rightarrow X$ there exists exactly one map $\bar{f}: A \rightarrow X_{\mathfrak{U}}$ such that

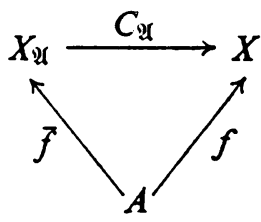

commutes.

In this case $C_{\mathfrak{Y}}: X_{\mathfrak{Y}} \rightarrow X$ is called the $\mathfrak{A}$-coreflection of $X$. It is uniquely determined by $\mathfrak{A}$ and $X$ up to homeomorphism, and $C_{\mathfrak{A}}$ maps $X_{\mathfrak{Q}}$ one-to-one onto $X$ [12].

Received by the editors May 28, 1968 and, in revised form, April 28, 1969.

( ${ }^{1}$ A subcategory $\mathfrak{U}$ of $\mathfrak{T}$ is full iff for two objects $A, B$ of $\mathfrak{I}$ the morphism sets $M \mathfrak{I}(A, B)$ and $M \mathfrak{I}(A, B)$ coincide.

${ }^{(2)}$ A subcategory $\mathfrak{A}$ or $\mathfrak{I}$ is replete (abstract) if $\mathfrak{I}$ contains with each space $A$ every space homeomorphic to $A$.

(3) Map = continuous function. 
So passing from $X$ to $X_{\mathfrak{I}}$ means nothing else than a modification of the topology of $X$ on the same underlying set.

$\mathfrak{A}$ is coreflective in $\mathfrak{I}$ iff $\mathfrak{A}$ is closed under the formation of disjoint topological unions and topological quotients [12]. If $\mathfrak{A}$ is any subcategory of $\mathfrak{T}$, the category $\mathfrak{A}$ of quotients of disjoint topological unions of members of $\mathfrak{A}$ is the smallest coreflective subcategory of $\mathfrak{I}$ containing $\mathfrak{A}[10]$. Therefore $\overline{\mathfrak{A}}$ is called the coreflective subcategory of $\mathfrak{T}$ generated by $\mathfrak{A}$. If we denote by

$$
\begin{aligned}
& \mathfrak{A}_{\text {sum }} \text { the category of all disjoint topological unions of members of } \mathfrak{A} \text {, } \\
& \mathfrak{A}_{\text {lok }} \quad \text { the category of all spaces } X \text { which are locally in } \mathfrak{A} \text { in the follow- } \\
& \text { ing sense: every point of } X \text { has a neighborhood belonging to } \mathfrak{A} \text {, } \\
& \mathfrak{A}_{\text {Gen }} \text { the category of all spaces } X \text { for which the closure of each subset } \\
& Y \text { consists of all points } x \in X \text { for which there exists a subspace } \\
& A \text { of } X \text { belonging to } \mathfrak{A} \text { with } x \in \mathrm{cl}_{A}(A \cap Y) \text {, } \\
& \mathfrak{A}_{\text {gen }} \text { the category of all spaces } X \text { which are } \mathfrak{A} \text {-generated in the } \\
& \text { following sense: a subset } Y \text { of } X \text { is closed in } X \text { iff its intersection } \\
& \text { with every subspace } A \text { of } X \text { belonging to } \mathfrak{A} \text { is closed in } A \text {, }
\end{aligned}
$$

then the following holds:

$$
\mathfrak{A} \subset \mathfrak{A}_{\text {sum }} \subset \mathfrak{A}_{\text {lok }} \subset \mathfrak{A}_{\text {Gen }} \subset \mathfrak{A}_{\text {gen }} \subset \overline{\mathfrak{A}} .
$$

If especially $\mathfrak{A}$ is coreflective in $\mathfrak{T}$ then

$$
\mathfrak{U}=\mathfrak{A}_{\text {sum }}=\mathfrak{A}_{\mathrm{lok}}=\mathfrak{A}_{\mathrm{Gen}}=\mathfrak{A}_{\text {gen }}=\overline{\mathfrak{A}} \text {. }
$$

The three main examples of coreflective subcategories of $\mathfrak{T}$ (the compactlygenerated spaces, the locally connected spaces, and the sequential spaces) suggest three special methods of generating coreflective subcategories of $\mathfrak{T}$ :

METHOD 1. If $\mathfrak{A}$ is a map-invariant topological property (i.e., a property preserved under continuous images) then $\mathfrak{A}_{\text {gen }}=\overline{\mathfrak{A}}$. If $\mathfrak{A}$ is the category of compact spaces $\mathfrak{A}_{\text {gen }}$ is the category of compactly generated spaces, if $\mathfrak{A}$ is the category of all finite spaces $\mathfrak{A}_{\text {gen }}$ is the category of all finitely-generated spaces, i.e., of all spaces $X$ with the property that any intersection of open subsets of $X$ is open in $X$. $\left(\mathfrak{A}_{\text {Gen }}\right.$ consists of all hereditary quotients of disjoint unions of spaces in $\mathfrak{A}$ [5]. A function $f: X \rightarrow Y$ with $X$ in $\mathfrak{A}_{\text {gen }}$ is continuous, if for every subspace $A$ of $X$ with $A$ in $\mathfrak{A}$ the restriction $f / A$ is continuous.)

METHOD 2. (a) If $\mathfrak{A}$ is map-invariant and has the property that the union of any centered family of subspaces of a space $X$ which are in $\mathfrak{A}$ is itself in $\mathfrak{A}$ (this ensures that each $X$ can be covered by pairwise disjoint maximal subspaces belonging to $\mathfrak{A}$, the $\mathfrak{A}$-components of $X$ ) then $\mathfrak{A}_{\text {sum }}=\mathfrak{A}_{\text {lok }}=\mathfrak{A}_{\text {Gen }}=\mathfrak{U}_{\text {gen }}=\overline{\mathfrak{U}}$.

(b) If $\mathfrak{A}$ is a category with the above mentioned properties then the category $\mathfrak{A}_{\text {Lok }}$ of all spaces with the property that the $\mathfrak{A}$-components of each open subset of $X$ are open in $X$ is coreflective in $\mathfrak{I}$. If $\mathfrak{A}$ consists of all connected spaces $\mathfrak{A}_{\text {sum }}$ consists of all disjoint unions of connected spaces, $\mathfrak{A}_{L_{\text {Lk }}}$ of all locally connected spaces. If $\mathfrak{A}$ consists of all pathwise-connected spaces, $\mathfrak{A}_{\text {sum }}$ consists of all disjoint unions 
of pathwise-connected spaces, $\mathfrak{A}_{\text {Lok }}$ of all locally pathwise-connected spaces. If $\mathfrak{A}$ consists of all one-point spaces, $\mathfrak{A}_{\text {sum }}=\mathfrak{A}_{\text {Lok }}$ consists of all discrete spaces. If $\mathfrak{A}$ consists of all indiscrete spaces, $\mathfrak{A}_{\text {sum }}=\mathfrak{A}_{\text {Lok }}$ consists of all disjoint unions of indiscrete spaces.

Whereas Method 1 (studied in [5], [10]) and Method 2 (studied in [10]) are too special to yield all coreflective subcategories of $\mathfrak{T}$, Method 3 inspired by the way in which sequential spaces are defined, enables us to obtain every coreflective subcategory of $\mathfrak{T}$. Moreover there is a one-to-one correspondence between coreflective subcategories in $\mathfrak{T}$ and idempotent limit-operators defined below:

METHOD 3. Let us consider an operator $l$ which associates with every pair $(X, A)$, where $X$ is a topological space and $A$ is a subset of $X$, another subset $l_{X} A$ of $X$, called the set of $l$-limit points of $A$ in $X . l$ is called a limit-operator iff $l$ satisfies the conditions (1), (2), and (3) below:

(1) If $A$ is a subset of $X$ then $A \subset l_{X} A \subset \bar{A}^{x}$.

(2) If $A$ and $B$ are subsets of $X$ then $l_{X}(A \cup B)=l_{X} A \cup l_{X} B$.

(3) If $f: X \rightarrow Y$ is a map and $A$ is a subset of $X$ then $f\left(l_{X} A\right) \subset l_{Y}(f A)$.

It is easily checked that any limit-operator $l$ satisfies the conditions (4) and (5) below:

(4) If $A$ and $B$ are subsets of $X$ then $A \subset B$ implies $l_{X} A \subset l_{X} B$.

(5) If $f: X \rightarrow Y$ is a map and $A$ is a subset of $Y$ with $l_{Y} A=A$ then $l_{X}\left(f^{-1} A\right)=f^{-1} A$.

An operator satisfying condition (5) is called a prelimit-operator. A limitoperator $l$ is called idempotent iff $l$ satisfies condition (6) below:

(6) If $A$ is a subset of $X$ then $l_{X}\left(l_{X} A\right)=l_{X} A$.

Proposition. If $l$ is a prelimit-operator the category $\mathfrak{A}(l)$ of all spaces $X$ with the property that each subset $A$ of $X$ with $l_{X} A=A$ is closed in $X$ turns out to be coreflective in $\mathfrak{T}$.

Proof. Let $X$ be a space which belongs to $\mathfrak{A}(l)$, let $f: X \rightarrow Y$ be a quotient-map. If $A$ is a subset of $Y$ with $l_{Y} A=A$ then (5) implies $l_{X}\left(f^{-1} A\right)=f^{-1} A$. Consequently $f^{-1} A$ is closed in $X$. Since $f$ is a quotient-map $A$ has to be closed in $Y$. So $Y$ belongs to $\mathfrak{A}(l)$. In an analogous manner one can show that $\mathfrak{A}(l)$ is closed under the formation not only of topological quotients but also of disjoint topological unions. This implies [12] that $\mathfrak{A}(l)$ is coreflective in $\mathfrak{T}$.

Proposition. If $l$ is a limit-operator then for each space $X$ the family

$$
\left\{A \mid A \subset X, l_{X} A=A\right\}
$$

is the family of all closed sets of a new topological space $X_{\mathfrak{U}}$ on the same underlying set as $X$ and the identity-map i from $X_{\mathfrak{Q}}$ into $X$ is the $\mathfrak{2}(l)$-coreflection of $X$.

Proof. The properties (1) and (2) of the limit-operator $l$ ensure that $X_{\mathfrak{A}}$ is a topological space and $i: X_{\mathfrak{U}} \rightarrow X$ is a continuous function. The properties (1) and (3) guarantee that $X_{\mathfrak{M}}$ belongs to $\mathfrak{A}(l)$. Finally condition (5) guarantees that any continuous function from a space $A$ belonging to $\mathfrak{Y}(l)$ into $X$ is continuous considered as a function from $A$ to $X_{\mathfrak{U}}$. 
Proposition. If $\mathfrak{A}$ is coreflective in $\mathfrak{T}$ and if we denote the coreflection-maps by $c_{\mathfrak{U}}: X_{\mathfrak{U}} \rightarrow X$ then $l_{X} A=c_{\mathfrak{U}}\left[\mathrm{cl}_{X_{\mathfrak{U}}}\left(c_{\mathfrak{U}}^{-1}[A]\right)\right]$ defines an idempotent limit-operator $l(\mathfrak{U})$.

Proof. Since $c_{2}$ is one-to-one and onto we can assume without loss of generality that $X_{\mathfrak{A}}$ and $X$ have the same underlying set and $c_{\mathfrak{A}}$ is the identity on this set. Then $l_{X} A=\bar{A}^{x_{\mathfrak{I}}}$. Conditions (1), (2), and (6) of an idempotent limit-operator are immediate consequences. Finally if $f: X \rightarrow Y$ is a map then $f$ can be regarded in a similar manner as a function from $X_{\mathfrak{U}}$ into $Y_{\mathfrak{U}}$ which is again continuous. This implies (3).

So with any prelimit-operator $l$ there is associated a coreflective subcategory $\mathfrak{A}(l)$ of $\mathfrak{I}$, and with any coreflective subcategory $\mathfrak{U}$ of $\mathfrak{I}$ there is associated an idempotent limit-operator $l(\mathfrak{A})$. This yields a one-to-one correspondence between idempotent limit-operators and coreflective subcategories of $\mathfrak{T}$ :

THEOREM. 1. If $l$ is an idempotent limit-operator then $l(\mathfrak{X}(l))=l$.

2. If $\mathfrak{A}$ is coreflective in $\mathfrak{T}$ then $\mathfrak{X}(l(\mathfrak{U}))=\mathfrak{U}$.

Moreover with any limit-operator $l$ there is an associated idempotent limit-operator $l=l(\mathfrak{A}(l))$. If the class of all limit-operators is ordered by $l \leqq l^{\prime}$ iff $l_{X} A \subset l_{X}^{\prime} A$ for every pair $(X, A) l$ turns out to be the smallest idempotent limit-operator $l^{\prime}$ with $l \leqq l^{\prime} . l$ can also be defined in the following way:

(a) $l_{x} A=\bigcap\left\{B \mid A \subset B \subset X, l_{x} B=B\right\}$.

(b) By transfinite induction: $l_{X}^{0} A=A, l_{X}^{\alpha} A=l\left(\bigcup_{\beta<\alpha} l_{X}^{\beta} A\right), l_{X} A=\bigcup_{\alpha} l_{X}^{\alpha} A$.

Making the class of all limit-operators into a category $\mathbb{S}$ by defining morphism-sets $M_{\mathfrak{C}}(l, k)$ in the following way: $M_{\mathbb{E}}(l, k)$ contains exactly one morphism iff $l \leqq k$ and is empty otherwise, we can state the above result as follows: the subcategory of all idempotent limit-operators is reflective in $\mathfrak{E}$. $l$ is the idempotent reflection of $l$.

REMARK. Many interesting theorems about sequential spaces arise from the fact that the sequential limit-operator is not idempotent. Most of these theorems can be generalized to arbitrary limit-operators.

EXAMPLES.

(1) $l_{X} A=X$ defines a prelimit-operator with $\mathfrak{A}(l)=\mathfrak{I}$.

(2) $l_{X} A=\bar{A}^{x}$ defines an idempotent limit-operator with $\mathfrak{X}(l)=\mathfrak{T}$.

(3) $l_{x} A=A$ defines an idempotent limit-operator. $\mathfrak{A}(l)$ is the category of all discrete spaces.

(4) $l_{X} A=\{x \in X \mid$ there exists an indiscrete subspace $S$ of $X$ with $x \in \operatorname{cl}(A \cap S)\}$ defines an idempotent limit-operator. $\mathfrak{A}(l)$ is the category of all topological unions of indiscrete spaces.

Proof. Obviously $l$ satisfies the conditions (1) and (2) of a limit-operator. Condition (3) follows from the fact that any continuous image of an indiscrete space is indiscrete, condition (6) from the fact that the union of any centered family of indiscrete subspaces of a space $X$ is an indiscrete subspace of $X$. Obviously any indiscrete space and hence any topological union of indiscrete spaces belongs to the coreflective subcategory $\mathfrak{A}(l)$. Let $X$ be a space and let $\left(X_{i}\right)$ be the family of all 
maximal indiscrete subspaces of $X$. Any $X_{i}$ is closed in $X$. If $X$ belongs to $\mathfrak{A}(l)$ any $X_{i}$ is open in $X$. Consequently $X$ is the topological union of the family $\left(X_{i}\right)$ of indiscrete spaces. In the following examples proofs are omitted.

(5) $l_{X} A=\{x \in X \mid$ there exists a constant sequence in $A$ converging to $X\}=$ $\{x \in X \mid$ there exists a finite subspace $F$ of $X$ with $x \in \operatorname{cl}(A \cap F)\}$ defines an idempotent limit-operator. $\mathfrak{U}(l)$ is the category of all spaces $X$ with the property that any intersection of open subsets of $X$ is open in $X$.

(6) $l_{X} A=\{x \in X \mid$ there exists a sequence in $A$ converging to $x\}$ defines a limitoperator which is not idempotent. $\mathfrak{U}(l)$ is the category of all sequential spaces, i.e. of all quotients of metrizable spaces [3].

(7) For each regular ordinal $\alpha$ an $\alpha$-sequence is a Moore-Smith sequence with the well-ordered index-set $W(\alpha)$ of all ordinals less than $\alpha . l_{X} A=\{x \in X \mid$ there exists an $\alpha$-sequence in $A$ converging to $x$ \} defines a limit-operator which is not idempotent. $\mathfrak{A}(l)$ is the category of all $\alpha$-sequential spaces.

(8) A well-ordered sequence ( $w$-sequence) is a Moore-Smith sequence with wellordered index-set. $l_{X} A=\{x \in X \mid$ there exists a $w$-sequence in $A$ converging to $X\}$ defines a limit-operator which is not idempotent. $\mathfrak{A}(l)$ is the category of all $w$ sequential spaces, i.e. of all quotients of orderable spaces [8].

(9) $l_{X} A=\{x \in X \mid$ there exists a compact subset $K$ of $X$ with $x \in \operatorname{cl}(A \cap K)\}$ defines a limit-operator which is not idempotent. $\mathfrak{A}(l)$ is the category of all compactly generated spaces.

(10) $l_{x} A=\{x \in X \mid$ there exists a connected subset $C$ of $X$ with $x \in \operatorname{cl}(A \cap C)\}$ defines a limit-operator which is not idempotent. $\mathfrak{A}(l)$ is the category of all disjoint unions of connected spaces.

(11) The property connected in (10), compact in (9), indiscrete in (4), finite in (5), can be replaced by any map-invariant property $\mathfrak{A}$.

Finally if $\mathfrak{A}$ is coreflective in $\mathfrak{T}$ the categories of all spaces which are open(closed-, clopen-)hereditary $\mathfrak{A}$ are coreflective in $\mathfrak{T}$.

II. The "lattice" $\mathfrak{c}$ of coreflective subcategories of $\mathfrak{T}$.

(1) $\mathfrak{A}_{1}$. $\mathfrak{C}$ contains a smallest element $\mathfrak{A}_{1}$, consisting of all discrete spaces. It can be generated by any nonempty discrete space.

(2) $\mathfrak{I}_{2} \cdot \mathfrak{E}-\{\mathfrak{Q}\}_{1}$ contains a smallest element $\mathfrak{U}_{2}$, consisting of all spaces $X$ with the following equivalent properties:

(a) $X$ is a disjoint union of indiscrete spaces,

(b) every open subset of $X$ is closed,

(c) every closed subset of $X$ is open,

(d) the $T_{0}$-reflection of $X$ is discrete.

$\mathfrak{U}_{2}$ can be generated by any indiscrete space with more than one point.

(3) $\mathfrak{A}_{3}$. $\mathfrak{E}-\left\{\mathfrak{Q}_{1}, \mathfrak{A}_{2}\right\}$ contains a smallest element $\mathfrak{H}_{3}$, consisting of all spaces $X$ with the following equivalent properties:

(a) $X$ is finitely generated (see $\S \mathrm{I}$, Method 1),

(b) for every $x \in X, A \subset X, x \in \bar{A}$ iff there exists $a \in A$ with $x \in\{\bar{\alpha}\}$, 
(c) every intersection of open subsets of $X$ is open in $X$,

(d) every union of closed subsets of $X$ is closed in $X$.

$\mathfrak{A}_{3}$ contains already all finite spaces. It can be generated by any space with two points and three open sets. $\mathfrak{A}_{3}$ is isomorphic to the category $\mathfrak{\Re}$ of preordered sets. Consequently $\mathfrak{B}$ is very small compared with $\mathfrak{T}$. It has only two proper coreflective subcategories namely $\mathfrak{P}_{1}$ consisting of all sets ordered by equality and $\mathfrak{P}_{2}$ consisting of all preordered sets whose order-reflections belong to $\mathfrak{P}_{1}$.

(4) $\mathfrak{B}_{\alpha}$. For every regular ordinal $\omega_{\alpha}$ let $X_{\alpha}$ be the set of all ordinals $\beta$ with $\beta \leqq \omega_{\alpha}$. If we consider a subset $B$ of $X_{\alpha}$ as open iff $B=\varnothing$ or there exists an ordinal $\beta$ with $\beta<\omega_{\alpha}$ and $B=\left\{\gamma \in X_{\alpha} \mid \beta \leqq \gamma\right\}$ then $X_{\alpha}$ becomes a topological space denoted by $B(\alpha)$. The coreflective subcategory $\mathfrak{B}_{\alpha}$ generated by $B(\alpha)$ is the smallest coreflective subcategory $\mathfrak{A}$ of $\mathfrak{I}$ with the following properties:

(1) if $X$ belongs to $\mathscr{A}$ then the intersection of less than $\aleph_{\alpha}$ open subsets of $X$ is open in $X$,

(2) $\mathfrak{A}$ contains a space $X$ having a family of $\boldsymbol{\aleph}_{\alpha}$ open subsets whose intersection is not open in $X$.

For every coreflective subcategory $\mathfrak{B}$ of $\mathfrak{I}$ with $\mathfrak{B} \notin\left\{\mathfrak{A}_{1}, \mathfrak{A}_{2}, \mathfrak{A}_{3}\right\}$ there exists a regular ordinal $\omega_{\alpha}$ with $\mathfrak{B}_{\alpha} \subset \mathfrak{B}$.

The $\mathfrak{B}_{\alpha}$ are pairwise not comparable since $\alpha<\beta$ implies:

1. $\mathfrak{B}_{\alpha} \subset \mathfrak{\complement}_{\alpha}$ (defined below) but $B(\beta) \notin \mathfrak{\bigvee}_{\alpha}$.

2. $\mathfrak{B}_{\beta} \subset \mathfrak{E}_{\beta}$ (defined below) but $B(\alpha) \notin \mathfrak{E}_{\beta}$.

Consequently the $\mathfrak{Q}_{\alpha}$ are the minimal elements of $\mathfrak{E}-\left\{\mathfrak{U}_{1}, \mathfrak{A}_{2}, \mathfrak{A}_{3}\right\}$.

(5) $\mathfrak{夭}_{\alpha}$. For every regular ordinal $\omega_{\alpha}$ let $\mathfrak{夭}_{\alpha}$ be the category of all $\omega_{\alpha}$-sequential spaces (cf. Example 7 above). $\mathbb{夭}_{\alpha}$ can be generated by the space $C(\alpha)$ defined below: let $X_{\alpha}$ be the set of all ordinals $\beta$ with $\beta \leqq \omega_{\alpha}$ and let a subset $B$ of $X_{\alpha}$ be open iff $\omega_{\alpha} \notin B$ or there exists an ordinal $\beta$ with $\beta<\omega_{\alpha}$ and $\left\{\gamma \in X_{\alpha} \mid \beta \leqq \gamma\right\} \subset B$. One thus obtains the space $C(\alpha)$. Obviously $\mathfrak{B}_{\alpha} \subset \mathfrak{C}_{\alpha}$ for every $\alpha$. Moreover $\mathfrak{B}_{\alpha} \neq \mathfrak{E}_{\alpha}$ since $C(\alpha)$ is not locally connected and $\mathfrak{B}_{\alpha}$ contains only locally connected spaces. The $\mathfrak{C}_{\alpha}$ are pairwise not comparable since $\alpha<\beta$ implies: 1. $C(\beta) \notin \mathfrak{E}_{\alpha}$. 2. $\mathfrak{E}_{\beta} \subset \mathfrak{E}_{\beta}$ (defined below) but $C(\alpha) \notin \mathfrak{E}_{\beta}$.

(6) $\mathcal{D}_{\alpha}$. For every regular ordinal $\omega_{\alpha}$ let $\mathscr{D}_{\alpha}$ denote the coreflective subcategory of $\mathfrak{T}$ generated by the disjoint topological union of all $C(\beta)$ with $\beta \leqq \omega_{\alpha}$. Obviously $\beta \leqq \alpha$ implies $\mathfrak{D}_{\beta} \subset \mathscr{D}_{\alpha}$ and $\mathfrak{D}=\bigcup_{\alpha} \mathfrak{D}_{\alpha}=\sup _{\alpha} \mathfrak{E}_{\alpha}$ is the category of all $w$-sequential spaces (cf. Example 8 above).

(7) $\mathfrak{E}_{\alpha}$. For every regular ordinal $\omega_{\alpha}$ let $\mathfrak{E}_{\alpha}$ be the category of all spaces $X$ with the property that every intersection of less than $\boldsymbol{\aleph}_{\alpha}$ open subsets of $X$ is open in $X$. Obviously $\mathfrak{E}_{\alpha} \subset \mathfrak{F}_{\alpha}$ for every $\alpha$, and $\mathfrak{E}_{\beta} \subset \mathfrak{F}_{\alpha}$ for $\alpha \leqq \beta$. The intersection of all $\mathfrak{E}_{\alpha}$ is $\mathfrak{A}_{3}$, the union of all $\mathfrak{F}_{\alpha}$ is $\mathbb{E}_{0}=\mathfrak{T}$.

(8) $\mathfrak{2}_{3}^{*}, \mathfrak{C}_{\alpha}^{*} . \mathfrak{2}_{3}^{*}$ is the category of all spaces $X$ with the following equivalent properties:

(a) every intersection of clopen subsets of $X$ is clopen in $X$,

(b) every union of clopen subsets of $X$ is clopen in $X$, 
(c) $X$ is a disjoint union of connected spaces,

(d) the totally disconnected reflection of $X$ is discrete.

Replacing open by clopen we can define in analogy to $\mathfrak{B}_{\alpha}$ and $\mathbb{E}_{\alpha}$ the categories $\mathfrak{B}_{\alpha}^{*}$ and $\mathfrak{F}_{\alpha}^{*}$. It turns out that $\mathfrak{B}_{\alpha}^{*}=\mathfrak{C}_{\alpha}$ for every $\alpha$. The intersection of all $\mathfrak{F}_{\alpha}^{*}$ is $\mathfrak{A}_{3}^{*}$.

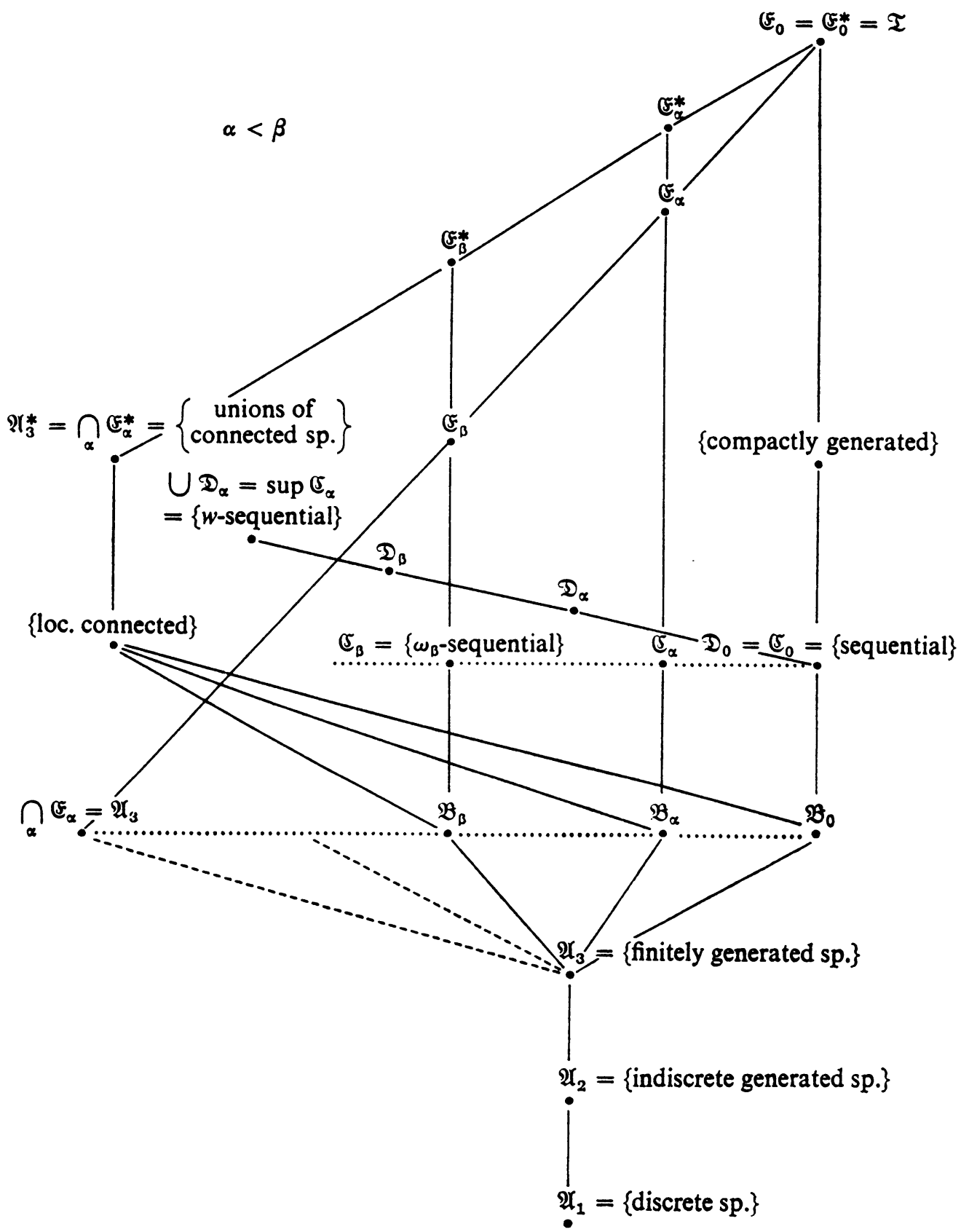




\section{LITERATURE}

1. A. B. Arhangelskii and S. P. Franklin, Ordinal invariants for topological spaces, Michigan Math. J. 15 (1968), 313-320.

2. S. Baron, The coreflective subcategory of sequential spaces (to appear).

3. S. P. Franklin, Spaces in which sequences suffice, Fund. Math. 57 (1965), 107-115.

4. - Spaces in which sequences suffice. II, Fund. Math. 61 (1967), 51-56.

5. - Natural covers (to appear).

6. - The categories of $k$-spaces and sequential spaces, Class notes, Carnegie Institute of Technology, Pittsburgh, Pa., 1967.

7. A. M. Gleason, Universal locally connected refinements, Illinois J. Math. 7 (1963), 521-531.

8. H. Herrlich, Quotienten geordneter Räume and Folgenkonvergenz, Fund. Math. 61 (1967), 79-81.

9. H. Herrlich and G. E. Strecker, Coreflective subcategories. I. Generalities (to appear).

10. - Coreflective subcategories. II. Applications in general topology (to appear).

11. H. Herrlich, Topologische Reflexionen und Coreflexionen, Lecture Notes in Math., No. 78, Springer-Verlag, Berlin, 1968.

12. J. F. Kennison, Reflective functors in general topology and elsewhere, Trans. Amer. Math. Soc. 118 (1965), 303-315.

13. G. S. Young, The introduction of local connectivity by change of topology, Amer. J. Math. 68 (1946), 479-494.

Freie UNIVERSitÄt BerLin, Federal Republic of Germany 